\title{
Sommaire de la Déclaration du Comité consultatif national de l'immunisation (CCNI) sur la vaccination antigrippale pour la saison 2021-2022
}

\author{
Angela Sinilaite ${ }^{1}$, Kelsey Young ${ }^{1}$, Robyn Harrison ${ }^{2,3}$ au nom du Comité consultatif national de \\ l'immunisation (CCNI)*
}

\section{Résumé}

Contexte : Plusieurs vaccins antigrippaux sont autorisés au Canada et les données probantes sur la vaccination contre la grippe sont en constante évolution. Le Comité consultatif national de l'immunisation (CCNI) présente chaque année à l'Agence de la santé publique du Canada (l'Agence) des recommandations concernant l'utilisation des vaccins contre la grippe saisonnière.

Objectifs : Résumer les recommandations du CCNI sur l'utilisation des vaccins antigrippaux pour la saison 2021-2022 et mettre en évidence les nouvelles recommandations.

Méthodes : Les recommandations annuelles concernant vaccination antigrippale sont élaborées par le Groupe de travail sur l'influenza du CCNI pour examen et approbation par le CCNI. L'élaboration des recommandations est basée sur le processus du CCNI fondé sur des données probantes.

Résultats : Les nouvelles recommandations suivantes ont été formulées : 1 ) Influvac ${ }^{\mathrm{MD}}$ Tetra peut être considéré comme une option parmi les vaccins antigrippaux inactivés quadrivalents à dose standard (IIV4-SD) proposés aux adultes et aux enfants âgés de trois ans et plus; 2) Fluzone ${ }^{\mathrm{MD}}$ Haute dose Quadrivalent (IIV4-HD) peut être considéré comme une option pour les personnes âgées de 65 ans et plus pour lesquelles il est actuellement recommandé de recevoir Fluzone ${ }^{\mathrm{MD}}$ Haute dose (trivalent); et 3) Flucelvax ${ }^{\mathrm{MD}}$ Quad peut être considéré comme I'un des vaccins grippaux quadrivalents proposés aux adultes et aux enfants âgés de neuf ans et plus pour la vaccination antigrippale annuelle. Des conseils pour l'utilisation des vaccins antigrippaux pendant la pandémie de coronavirus de 2019 sont également soulignés.

Conclusion : Le CCNI continue de recommander qu'un vaccin antigrippal indiqué selon l'âge soit proposé chaque année à toute personne de six mois et plus qui ne présente aucune contre-indication à l'administration du vaccin. La vaccination doit être proposée en priorité aux personnes présentant un risque élevé de complications liées à la grippe ou d'hospitalisation, aux personnes susceptibles de transmettre la grippe aux personnes présentant un risque élevé de complications, et à d'autres personnes, selon les indications.
Cette oeuvre est mise à la disposition selon les termes de la licence internationale Creative Commons Attribution 4.0

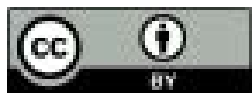

Affiliations

${ }^{1}$ Centre de l'immunisation et des maladies respiratoires infectieuses, Agence de la santé publique du Canada, Ottawa, ON

2 Présidente du Groupe de travail sur l'influenza du CCNI

${ }^{3}$ Université de l'Alberta, Alberta Health Services, Edmonton, AB

\section{*Correspondance :}

naci-ccni@phac-aspc.gc.ca

Citation proposée : Sinilaite A, Young K, Harrison R, au nom du Comité consultatif national de l'immunisation (CCNI). Sommaire de la Déclaration du Comité consultatif national de l'immunisation (CCNI) sur la vaccination antigrippale pour la saison 2021-2022. Relevé des maladies transmissibles au Canada 2021;47(9):411-20. https://doi.org/10.14745/ccdr.v47i09a04f

Mots-clés : Comité consultatif national de l'immunisation, CCNI, influenza, grippe, vaccin antigrippal, conseils

\section{Introduction}

La grippe saisonnière est une maladie virale infectieuse qui sévit dans le monde entier et dont le taux d'attaque annuel est estimé à $5 \%$ entre $10 \%$ chez les adultes et à $20 \%$ entre $30 \%$ chez les enfants (1). Des épidémies de grippe saisonnière surviennent chaque année au Canada, généralement à la fin de l'automne et en hiver; toutefois, le fardeau de la grippe peut varier d'une 
année à l'autre. On peut trouver des renseignements à jour sur l'activité grippale à l'échelle mondiale sur le site Web FluNet de l'Organisation mondiale de la santé (2) et à l'échelle nationale sur le site Web ÉpiGrippe de l'Agence de la santé publique du Canada (l'Agence) (3).

Le Comité consultatif national de l'immunisation (CCNI) présente à l'Agence des recommandations annuelles concernant I'utilisation des vaccins contre la grippe saisonnière, qui tiennent compte des changements ciblés dans l'épidémiologie de la grippe, des pratiques d'immunisation et des produits de vaccination antigrippale autorisés au Canada. L'élaboration des recommandations annuelles sur la vaccination antigrippale, qui est dirigée par le Groupe de travail sur l'influenza du CCNI, implique un examen et une évaluation approfondis de la littérature ainsi que des discussions et des débats scientifiques et de pratique clinique sur une variété de questions, qui peuvent inclure les suivantes : le fardeau de la maladie grippale et les populations ciblées par la vaccination, l'efficacité potentielle, l'efficacité réelle l'immunogénicité et la sécurité des vaccins antigrippaux, les calendriers de vaccination et d'autres aspects de la vaccination antigrippale. Les questions liées à l'éthique, à l'équité, à la faisabilité et à l'acceptabilité sont également examinées systématiquement par le CCNI pour l'élaboration complète des directives relatives aux vaccins (4).

L'objectif du présent article est de fournir un résumé des recommandations du CCNI et des renseignements à l'appui pour la saison grippale 2021-2022, y compris les conclusions des examens des preuves sur 1) un nouveau vaccin quadrivalent inactivé contre l'influenza biosimilaire, à base d'œufs (Influvac ${ }^{\mathrm{MD}}$ Tetra; VII4-SD), 2) un nouveau vaccin quadrivalent inactivé contre I'influenza à haute dose, à base d'œufs (Fluzone ${ }^{\mathrm{MD}}$ Haute dose Quadrivalent; VII4-HD), et 3) un vaccin contre l'influenza, à base de culture de cellules de mammifères (FlucelvaxMD Quad; VII4-cc). Vous trouverez tous les détails sur le site Web de I'Agence dans la Déclaration du comité consultatif du CCNI : Chapitre sur la grippe du Guide canadien d'immunisation et Déclaration sur la vaccination antigrippale pour la saison (la Déclaration) (5) et publications connexes.

\section{Abréviations des vaccins antigrippaux}

Les abréviations à jour utilisées par le CCNI pour décrire les caractéristiques des différents types de vaccins antigrippaux sont présentées dans le tableau 1.

\section{Méthodes}

Lors de la préparation des recommandations sur la vaccination antigrippale pour la saison 2021-2022, le Groupe de travail sur l'influenza du CCNI a reconnu la nécessité de procéder à un examen des données probantes relatives aux nouvelles thématiques, puis a examiné et analysé les données probantes disponibles et présenté des recommandations nouvelles ou

\section{Tableau 1 : Abréviations des vaccins antigrippaux employées par le CCNI}

\begin{tabular}{|c|c|c|c|}
\hline $\begin{array}{c}\text { Catégorie } \\
\text { de vaccin } \\
\text { contre } \\
\text { l'influenza }\end{array}$ & Formulation & Type & $\begin{array}{c}\text { Nouvelle } \\
\text { abréviation du } \\
\text { CCNIa }\end{array}$ \\
\hline \multirow{6}{*}{$\begin{array}{l}\text { Vaccin } \\
\text { inactivé } \\
\text { contre } \\
\text { l'influenza } \\
\text { (VII) }\end{array}$} & \multirow{3}{*}{$\begin{array}{l}\text { Trivalent } \\
\text { (VII3) }\end{array}$} & $\begin{array}{l}\text { À dose standard }{ }^{\mathrm{b}} \text {, } \\
\text { sans adjuvant, } \\
\text { administré par } \\
\text { voie IM, à base } \\
\text { d'œufs }^{\prime}\end{array}$ & VII3-SD \\
\hline & & $\begin{array}{l}\text { Avec adjuvantc, } \\
\text { administré par } \\
\text { voie IM, à base } \\
\text { d'œufs }\end{array}$ & VII3-adj \\
\hline & & $\begin{array}{l}\text { À haute dosed, } \\
\text { sans adjuvant, } \\
\text { administré par } \\
\text { voie IM, à base } \\
\text { d'œufs }\end{array}$ & VII3-HD \\
\hline & \multirow{3}{*}{$\begin{array}{l}\text { Quadrivalent } \\
\text { (VII4) }\end{array}$} & $\begin{array}{l}\text { À dose standard }{ }^{\mathrm{b}} \text {, } \\
\text { sans adjuvant, } \\
\text { administré par } \\
\text { voie IM, à base } \\
\text { d'œufs }^{\text {'cul }}\end{array}$ & VII4-SD \\
\hline & & $\begin{array}{l}\text { À dose standard }{ }^{\mathrm{b}} \text {, } \\
\text { sans adjuvant, } \\
\text { administré par } \\
\text { voie IM, basée sur } \\
\text { culture cellulaire }\end{array}$ & VII4-cc \\
\hline & & $\begin{array}{l}\text { À haute dosed, } \\
\text { sans adjuvant, } \\
\text { administré par } \\
\text { voie IM, à base } \\
\text { d'œufs }\end{array}$ & VII4-HD \\
\hline \multirow{2}{*}{$\begin{array}{l}\text { Vaccin vivant } \\
\text { atténué } \\
\text { contre } \\
\text { l'influenza } \\
\text { (WVAl) }\end{array}$} & $\begin{array}{l}\text { Trivalent } \\
\text { (WVAI3) }\end{array}$ & $\begin{array}{l}\text { Sans adjuvant, } \\
\text { vaporisateur } \\
\text { nasal, à base } \\
\text { d'œufs }\end{array}$ & WAI3 \\
\hline & $\begin{array}{l}\text { Quadrivalent } \\
\text { (VNAI4) }\end{array}$ & $\begin{array}{l}\text { Sans adjuvant, } \\
\text { vaporisateur } \\
\text { nasal, à base } \\
\text { d'œufs }\end{array}$ & WVAI4 \\
\hline
\end{tabular}

Abréviations : CCNI, Comité consultatif national de l'immunisation; IM, intramusculaire; VII vaccin inactivé contre l'influenza; VII3, vaccin trivalent inactivé contre l'influenza; VII3-Adj, vaccin trivalent inactivé contre l'influenza avec adjuvant à base d'œufs; VII3-HD, vaccin trivalent inactivé contre l'influenza à haute dose à base d'œufs; VII3-SD, vaccin trivalent inactivé contre l'influenza contre l'influenza à haute dose à base d'œufs; VIII-SD, vaccin trivalent inactivé contre I'influenza
à dose standard à base d'œufs; VII4, vaccin quadrivalent inactivé contre I'influenza; VII4-cc, vaccin quadrivalent inactivé contre l'influenza à dose standard à base de culture de cellules; VII4-HD, vaccin quadrivalent inactivé contre l'influenza à haute dose à base d'œufs; VII4-SD, vaccin quadrivalent inactivé contre l'influenza à dose standard à base d'œufs; VVAl, vaccin vivant atténué contre l'influenza; WAI3, vaccin trivalent atténué contre l'influenza à base d'œufs; VAI4, vaccin quadrivalent vivant atténué contre l'influenza à base d'œufs

a Le suffixe numérique correspond au nombre d'antigènes contenus dans le vaccin (le chiffre " 3 » renvoie à la formulation trivalente et le chiffre « 4 », à la formulation quadrivalente). Le suffixe composé «-SD » renvoie aux produits VII sans adjuvant qui contiennent $15 \mu \mathrm{g}$ d'HA par souche et qui sont administrés par voie intramusculaire en dose de $0,5 \mathrm{~mL}$; «-cc » renvoie à un VII fabriqué à partir du virus de la grippe cultivé dans des cultures cellulaires au lieu d'œufs de poule (Flucelvax ${ }^{\mathrm{MD}} \mathrm{Quad}$ ); " -Adj » renvoie à un VII avec adjuvant (VII3-Adj pour Fluad ${ }^{\mathrm{MD}}$ ou Fluad Pédiatrique ${ }^{\mathrm{MD}}$ ); et « $-\mathrm{HD}$ » renvoie à un $\mathrm{VII}$ dont la quantité d'antigènes est supérieure à $15 \mu \mathrm{g}$ de HA par souche (VII3-HD pour Fluzone ${ }^{\mathrm{MD}}$ Haute dose ou VII4-HD pour Fluzone ${ }^{\circledast}$ Haute

dose Quadrivalent)

c 7,5 $\mu \mathrm{g}$ (dans $0,25 \mathrm{~mL}$ ) ou $15 \mu \mathrm{g}$ (dans $0,5 \mathrm{~mL}$ ) de HA par souchen

d $60 \mu \mathrm{g}$ de HA par souche

Source : Tableau tiré de la Déclaration du CCNI sur la vaccination antigrippale pour la saison 2021-2022 (5) 
actualisées selon le processus d'élaboration de recommandations fondé sur les données probantes du CCNI (6). Pour une explication plus détaillée de la force des recommandations du $\mathrm{CCNI}$ et de la classification des preuves, consultez le tableau A1 de l'annexe. Un cadre publié, revu par des pairs, et des outils fondés sur des données probantes (notamment les filtres d'éthique intégrés, la matrice d'équité, la matrice de faisabilité et la matrice d'acceptabilité) ont été appliqués pour garantir que les questions liées à l'éthique, à l'équité, à la faisabilité et à l'acceptabilité étaient systématiquement évaluées et intégrées dans les directives (4).

Pour la saison grippale 2021-2022, le Groupe de travail sur I'influenza a examiné les preuves concernant l'utilisation de deux nouveaux vaccins : 1) Influvac Tetra, un nouveau vaccin quadrivalent inactivé contre l'influenza biosimilaire, à base d'œufs; et 2) Fluzone Haute dose (HD) Quadrivalent, un vaccin quadrivalent inactivé contre l'influenza à haute dose, à base d'œufs (VII4). L'utilisation d'Influvac Tetra (VII4-SD) a été autorisée pour la première fois au Canada chez les adultes en mars 2019, puis chez les enfants âgés de trois ans et plus en février 2020. Fluzone Haute dose (HD) Quadrivalent a été autorisé pour la première fois au Canada chez les adultes en juin 2020. Une formulation trivalente, Fluzone Haute dose, était auparavant autorisée pour une utilisation chez les adultes de 65 ans et plus au Canada, et recommandée par le CCNI, mais la commercialisation de ce vaccin a été interrompue en février 2021. Suite à l'examen et à l'analyse des données d'essais cliniques préalable à l'homologation disponibles et des rapports d'examen clinique de Santé Canada pour ces deux vaccins, le Groupe de travail sur l'influenza a proposé au CCNI de nouvelles recommandations pour l'utilisation des vaccins. Le CCNI a procédé à une évaluation critique des preuves disponibles et a approuvé les recommandations précises présentées.

Les recommandations et les preuves à l'appui de l'utilisation du vaccin inactivé contre la grippe saisonnière issu de culture de cellules de mammifères (Flucelvax Quad) provenant de la Déclaration supplémentaire du CCNI - Vaccins antigrippaux issus de cultures cellulaires de mammifères (7) ont également été intégrées à la Déclaration sur la vaccination antigrippale pour la saison 2021-2022. Flucelvax Quad est le premier et le seul vaccin inactivé contre la grippe saisonnière issu de culture cellulaire de mammifères disponible au Canada; il a été autorisé pour la première fois chez les adultes et les enfants âgés de neuf ans et plus le 22 novembre 2019. Le Groupe de travail sur l'influenza a supervisé l'étude systématique visant le développement de directives sur l'utilisation de Flucelvax Quad (VII4-cc). Six bases de données électroniques (EMBASE, MEDLINE, Scopus, ProQuest Public Health et ClinicalTrials.gov) ont été consultées depuis la création jusqu'au 12 février 2019, à l'aide d'une stratégie de recherche prédéfinie, afin de cibler la littérature pertinente sur l'efficacité potentielle, l'efficacité réelle, l'immunogénicité et l'innocuité chez les adultes et les enfants âgés de quatre ans et plus. Les essais cliniques enregistrés et la littérature grise des autorités internationales de santé publique et des groupes consultatifs techniques nationaux sur la vaccination ont également été pris en compte. En outre, un examinateur a effectué une recherche manuelle dans les listes de références des articles inclus afin de trouver d'autres publications pertinentes. Deux examinateurs ont passé en revue de manière indépendante les titres et les résumés des dossiers extraits de la recherche et les articles en texte intégral admissibles pour inclusion. Un examinateur a extrait les données des études admissibles et a évalué la qualité méthodologique de ces études à l'aide des critères définis par Harris et al. (8). Un deuxième examinateur a validé de manière indépendante l'extraction des données et l'évaluation de la qualité. Une synthèse descriptive des données extraites a été réalisée. Le CCNI a fourni de nouvelles recommandations basées sur l'évaluation des preuves.

\section{Résultats}

\section{Utilisation du vaccin contre la grippe saisonnière en présence du coronavirus 2019}

Compte tenu de la pandémie actuelle de maladie à coronavirus 2019 (COVID-19), l'Agence, en consultation avec le CCNI et le Comité canadien sur l'immunisation, a élaboré les lignes directrices supplémentaires suivantes sur la prestation des programmes de vaccination contre la grippe et l'administration du vaccin contre la grippe saisonnière afin d'appuyer les programmes de vaccination provinciaux et territoriaux et les fournisseurs de soins primaires pendant la pandémie de COVID-19 pour 2021-2022 :

- $\quad$ Lignes directrices sur l'administration de vaccins contre la grippe en présence de la COVID-19 (9)

- Lignes directrices sur l'utilisation de vaccins contre la grippe en présence de la COVID-19 (10)

Ces lignes directrices sont fondées sur les preuves scientifiques et les avis d'experts actuellement disponibles. Le contenu sera passé en revue régulièrement et des mises à jour seront effectuées si nécessaire tout au long de la prochaine saison de grippe, au fur et à mesure que le contexte de la santé publique évolue et que de nouvelles preuves et questions politiques apparaissent.

\section{Nouveau vaccin quadrivalent contre l'influenza à base d'œufs}

Le CCNI a conclu que I'Influvac Tetra est sûr et que son immunogénicité n'est pas inférieure à celle de la formulation trivalente de l'Influvac. Par conséquent, le CCNI a recommandé que I'Influvac Tetra puisse être considéré comme faisant partie des vaccins inactivés quadrivalents contre l'influenza à dose standard (VII4-SD) proposés aux adultes et aux enfants âgés de trois ans et plus (recommandation discrétionnaire du CCNI). 


\section{Nouveau vaccin quadrivalent contre l'influenza à base d'œufs à haute dose}

Le CCNI a conclu que Fluzone Haute dose Quadrivalent est comparable en termes de sécurité et d'immunogénicité à la formulation trivalente de Fluzone Haute dose précédemment autorisée. Par conséquent, le CCNI a émis la recommandation discrétionnaire suivante au niveau individuel sur l'utilisation de Fluzone Haute Dose Quadrivalent (VII4-HD) : Pour les personnes âgées de 65 ans et plus pour lesquelles il est actuellement recommandé de recevoir Fluzone Haute dose (trivalent), le CCNI recommande que Fluzone Haute dose Quadrivalent (VII4-HD) puisse être considéré comme une option (recommandation discrétionnaire du CCNI). Les recommandations pour les programmes de santé publique restent inchangées à ce jour.

\section{Inclusion d'un vaccin quadrivalent contre l'influenza à base de culture de cellules de mammifères}

Les données publiées revu par des pairs sur l'efficacité réelle, l'immunogénicité et l'innocuité des VII4-cc fabriqués à partir de virus entièrement dérivés de cellules étaient rares. L'examen systématique a permis de recenser quatre études d'observation (11-14) portant sur l'efficacité réelle du vaccin VII4-cc par rapport au vaccin VII à base d'œufs et deux essais contrôlés randomisés revu par des pairs portant sur I'immunogénicité et l'innocuité du vaccin VII4-cc par rapport à différentes formulations de VII3-cc (produites selon le même procédé de fabrication basé sur la culture de cellules rénales canines Madin-Darby [MDCK]). Des données probantes indiquent que le vaccin VII4-cc pourrait être plus efficace que les vaccins antigrippaux VII3 et VII4 à base d'œufs contre les résultats non confirmés en laboratoire liés à la grippe, y compris les interactions avec les services de santé liées à la grippe et le syndrome grippal. Bien que certaines données suggèrent que le vaccin VII4-cc puisse être plus efficace contre l'infection par le virus de la grippe $A(H 3 N 2)$ confirmée en laboratoire que le vaccin VII à base d'œufs, aucune différence d'efficacité réelle cohérente et statistiquement significative n'a été déterminée chez les adultes ou les enfants vaccinés avec le vaccin VII4-cc par rapport au vaccin VII à base d'œufs. Deux études évaluant I'immunogénicité et l'innocuité de VII4-cc par rapport à différentes formulations de VII3-cc (produites par Seqirus selon le même procédé de fabrication basé sur la culture de cellules MDCK) ont été ciblées dans cet examen $(15,16)$. Des données probantes indiquent également que le profil d'immunogénicité et d'innocuité de VII4-cc est comparable à celui des vaccins antigrippaux à base d'œufs déjà homologués au Canada et à la formulation trivalente de ce vaccin antigrippal à base de culture cellulaire qui a été homologué aux États-Unis et en Europe, mais pour lequel on n'a jamais demandé d'homologation au Canada (17-22).
Sur la base de l'évaluation des essais cliniques et des données d'observation disponibles avant et après la mise sur le marché, le CCNI a conclu que le VII-cc est une alternative efficace, sûre, bien tolérée et immunogène aux vaccins antigrippaux classiques à base d'œufs pour les enfants et les adultes. Par conséquent, le CCNI a formulé la recommandation suivante, qui complète la recommandation générale du CCNI concernant la vaccination contre la grippe, disponible dans la Déclaration du CCNI sur la vaccination antigrippale pour la saison (5):

Le CCNI recommande que Flucelvax Quad puisse être considéré parmi les VII4 proposés aux adultes et aux enfants de neuf ans et plus (recommandation discrétionnaire du CCNI).

- Le CCNI conclut qu'il existe des preuves suffisantes pour recommander la vaccination des adultes et des enfants âgés de neuf ans et plus avec Flucelvax Quad (niveau de preuve B)

Pour obtenir des détails complets sur cet examen, la justification, les considérations pertinentes et des renseignements supplémentaires à l'appui de cette recommandation, veuillez vous reporter à la Déclaration supplémentaire du CCNI : Vaccins antigrippaux issus de cultures cellulaires de mammifères (7). Notamment, Flucelvax Quad a récemment été autorisé par Santé Canada pour une utilisation chez les adultes et les enfants âgés de deux ans et plus. Cette indication d'âge autorisée actualisée remplace les renseignements relatifs à Flucelvax Quad figurant dans les sections pertinentes de la Déclaration du CCNI sur la vaccination antigrippale pour la saison 2021-2022 (5). De plus amples renseignements sont disponibles dans la nouvelle monographie de produit pour ce vaccin (23).

\section{Résumé des recommandations du Comité consultatif national de I'immunisation sur l'utilisation des vaccins antigrippaux pour la saison 2021-2022}

Le CCNI continue de recommander la vaccination antigrippale à toute personne âgée de six mois et plus qui ne présente aucune contre-indication à l'administration du vaccin. La vaccination devrait être proposée en priorité aux personnes qui présentent un risque élevé de complications ou d’hospitalisation liées à la grippe, aux personnes susceptibles de transmettre la grippe à des sujets à risque élevé de complications, et à d'autres personnes comme l'illustre la liste 1.

Les recommandations concernant le choix du vaccin antigrippal par groupe d'âge et par posologie et voie d'administration selon l'âge sont résumées aux tableau 2 et tableau 3, respectivement. 


\section{Liste 1 : Groupes pour lesquels le vaccin antigrippal est particulièrement recommandé}

Personnes présentant un risque élevé de complications ou d'hospitalisation liées à la grippe

- Tous les enfants âgés de 6 à 59 mois

- $\quad$ Adultes et enfants atteints d'une des affections chroniques suivantes ${ }^{\mathrm{a}}$ :

- Maladies cardiaques ou pulmonaires (notamment dysplasie bronchopulmonaire, fibrose kystique et asthme)

- Diabète sucré et autres maladies métaboliques

- Cancer, troubles liés à l'immunodépression (résultant d'une maladie sous-jacente, d'un traitement, ou des deux, par exemple la greffe d'un organe plein ou de cellules souches hématopoïétiques)

- Néphropathie

- Anémie ou hémoglobinopathie

- Troubles neurologiques ou du développement neurologique (ces troubles comprennent les troubles neuromusculaires, neurovasculaires, neurodégénératifs et du développement neurologique ainsi que les troubles convulsifs [et, pour les enfants, les convulsions fébriles et les retards de développement isolés], mais ils excluent les migraines et les troubles psychiatriques sans troubles neurologiques)

- Obésité morbide (indice de masse corporelle [IMC] de 40 ou plus)

- Enfants et adolescents (de 6 mois à 18 ans) sous traitement pendant de longues périodes par de l'acide acétylsalicylique, en raison de la possibilité d'un risque accru de syndrome de Reye associé à la grippe

- Toutes les femmes enceintes

- Résidents de maisons de soins infirmiers et d'autres établissements de soins de longue durée, quel que soit leur âge

- $\quad$ Personnes de 65 ans et plus

- Autochtones

Personnes qui pourraient transmettre la grippe à des sujets à risque élevé

- Travailleurs de la santé et autres fournisseurs de soins dans des établissements et en milieux communautaires qui, par leurs activités, pourraient transmettre la grippe à des sujets à risque élevé de complications

- Contacts familiaux (adultes et enfants) de personnes à risque élevé de complications liées à la grippe, que ces dernières aient été vaccinées ou non :

- Contacts familiaux de personnes à risque élevé

- Contacts familiaux des nourrissons âgés de moins de 6 mois, qui sont à risque élevé de complications grippales, mais qui ne peuvent pas recevoir un vaccin contre la grippe

- Membres d'un ménage devant accueillir un nouveau-né durant la saison grippale

- Personnes qui s'occupent régulièrement d'enfants de 0 à 59 mois, que ce soit à la maison ou à l'extérieur

- Personnes qui fournissent des services à des sujets à risque élevé dans un milieu fermé ou relativement fermé (e.g. équipage de navire)

Autres

- Personnes qui fournissent des services communautaires essentiels

- Personnes en contact direct avec de la volaille infectée par le virus de la grippe aviaire durant les activités d'abattage

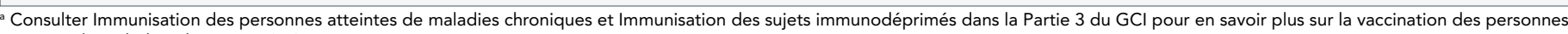
atteintes de maladies chroniques (24)

Source : Liste tirée de la Déclaration du CCNI sur la vaccination antigrippale pour la saison 2021-2022 (5)

\section{Tableau 2 : Recommandations concernant le choix du vaccin antigrippale pour le processus décisionnel à l'échelle individuelle et des programmes de santé publique, par groupe d'âge}

\begin{tabular}{|c|c|c|}
\hline $\begin{array}{c}\text { Groupe } \\
\text { d'âge }\end{array}$ & $\begin{array}{l}\text { Types de } \\
\text { vaccins } \\
\text { autorisés }\end{array}$ & Recommandations concernant le choix du vaccin antigrippal \\
\hline 6 à 23 mois & $\begin{array}{l}\text { VII3-SDa } \\
\text { VII3-Adj } \\
\text { VII4-SD }\end{array}$ & $\begin{array}{l}\text { - Un vaccin antigrippal quadrivalent homologué pour ce groupe d'âge devrait être utilisé pour les nourrissons et les } \\
\text { jeunes enfants ne présentant pas de contre-indications, compte tenu du fardeau du virus de la grippe B dans ce } \\
\text { groupe d'âge et de la possibilité de non-concordance entre la souche prédominante du virus de la grippe B en } \\
\text { circulation et la souche d'un vaccin trivalent. } \\
\text { - En l'absence d'un vaccin quadrivalent, l'un ou l'autre des vaccins trivalents homologués doit être utilisé pour ce } \\
\text { groupe d'âge. }\end{array}$ \\
\hline 2 à $17 a^{a n s}$ & $\begin{array}{l}\text { VII3-SD } \\
\text { VII4-SD } \\
\text { VII4-cc } \\
\text { (9 ans et plus) } \\
\text { VVAI4 }\end{array}$ & 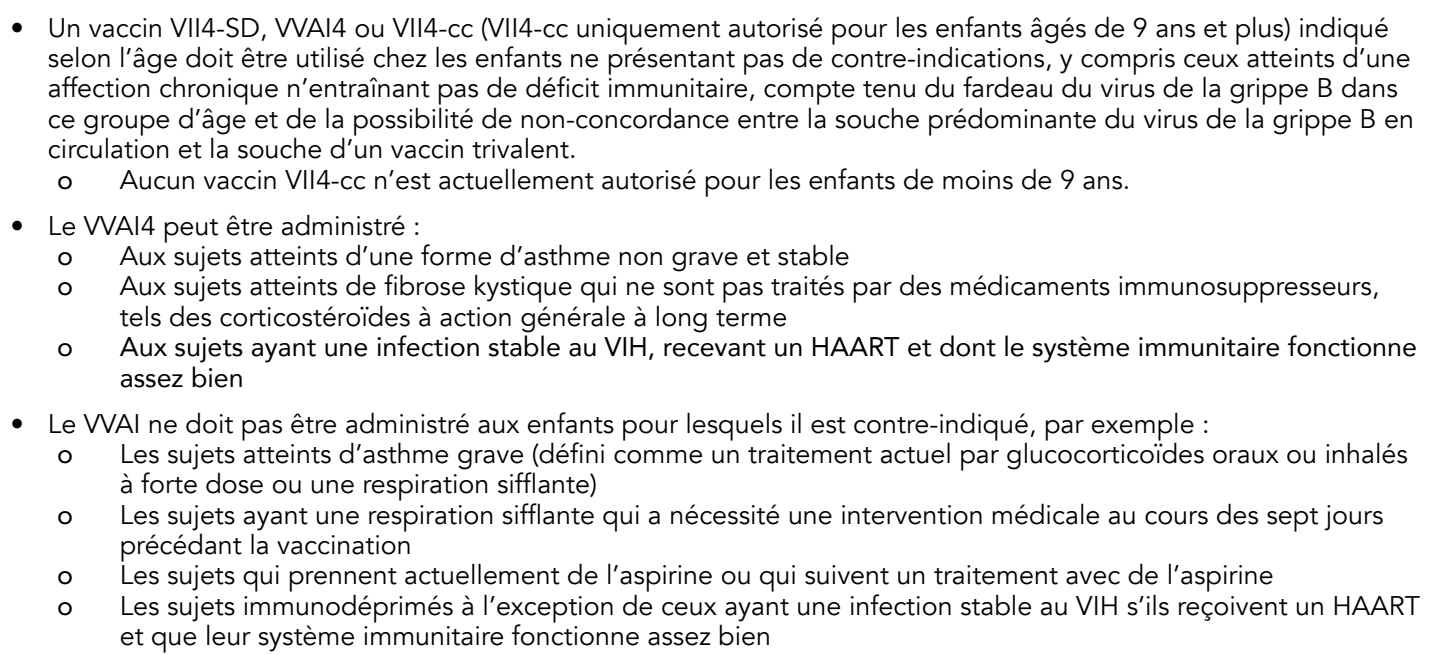 \\
\hline
\end{tabular}


Tableau 2 : Recommandations concernant le choix du vaccin antigrippale pour le processus décisionnel à l'échelle individuelle et des programmes de santé publique, par groupe d'âge (suite)

\begin{tabular}{|c|c|c|c|}
\hline $\begin{array}{l}\text { Groupe } \\
\text { d'âge }\end{array}$ & $\begin{array}{l}\text { Types de } \\
\text { vaccins } \\
\text { autorisés }\end{array}$ & \multicolumn{2}{|c|}{ Recommandations concernant le choix du vaccin antigrippal } \\
\hline $\begin{array}{l}2 \text { à } 17 \text { ans }^{b} \\
\text { (suite) }\end{array}$ & $\begin{array}{l}\text { VII3-SD } \\
\text { VII4-SD } \\
\text { VII4-cc } \\
\text { (9 ans et plus) } \\
\text { VVAI4 (suite) }\end{array}$ & \multicolumn{2}{|c|}{$\begin{array}{l}\text { - Le VVAI est contre-indiqué pour les adolescentes enceintes. Le VII4-SD ou VII4-ccc doivent être utilisés à la place. } \\
\text { - Si le VII4-SD, le VII4-cc }{ }^{c} \text { et le VVAI4 ne sont pas disponibles, le VII3-SD doit être utilisé. }\end{array}$} \\
\hline 18 à 59 ans & $\begin{array}{l}\text { VII3-SD } \\
\text { VII4-SD } \\
\text { VII4-cC } \\
\text { VVAI4 }\end{array}$ & \multicolumn{2}{|c|}{$\begin{array}{l}\text { - Tous les vaccins antigrippaux disponibles peuvent être utilisés chez les adultes qui n'ont pas de contre-indications. } \\
\text { - L II existe des preuves que le VII peut être plus efficace que le VVAl chez les adultes en bonne santé } \\
\text { - Le VVAI n'est pas recommandé pour les personnes suivantes : } \\
\text { o Femmes enceintes } \\
\text { ○ Adultes souffrant de l'une des affections chroniques énumérées dans la liste 1, y compris les affections } \\
\text { immunodépressives } \\
\text { - Travailleurs de la santé }\end{array}$} \\
\hline 60 à 64 ans & $\begin{array}{l}\text { VII3-SDa } \\
\text { VII4-SD } \\
\text { VII4-cc }\end{array}$ & \multicolumn{2}{|c|}{ - Tous les vaccins antigrippaux disponibles peuvent être utilisés chez ceux qui n'ont pas de contre-indications. } \\
\hline \multirow[t]{2}{*}{$\begin{array}{l}65 \text { ans et } \\
\text { plus }^{d}\end{array}$} & $\begin{array}{l}\text { VII3-SDa } \\
\text { VII3-Adj }\end{array}$ & Prise de décision sur le plan individuel & $\begin{array}{c}\text { Prise de décision sur le plan des programmes de santé } \\
\text { publique }\end{array}$ \\
\hline & $\begin{array}{l}\text { VII3-HDe } \\
\text { VII4-SD } \\
\text { VII4-cc }\end{array}$ & $\begin{array}{l}\text { - Le VII-HD devrait être utilisé plutôt que le VII- } \\
\text { SD, compte tenu du fardeau de la maladie } \\
\text { associée à la grippe A(H3N2) et des données } \\
\text { probantes suffisantes à l'appui d'une meilleure } \\
\text { protection du VII3-HD par rapport au VII3-SD } \\
\text { chez les adultes de } 65 \text { ans et plus. } \\
\text { o Hormis la recommandation d'utiliser } \\
\text { les formulations VII-HD plutôt que } \\
\text { VII-SD, le CCNI n'a pas formulé de } \\
\text { recommandations comparatives sur le } \\
\text { plan individuel sur l'utilisation des autres } \\
\text { vaccins disponibles dans ce groupe } \\
\text { d'âge. En l'absence d'un produit précis, } \\
\text { il convient d'utiliser l'un des vaccins } \\
\text { antigrippaux disponibles adaptés à l'âge. }\end{array}$ & $\begin{array}{l}\text { - N'importe lequel des vaccins antigrippaux disponibles } \\
\text { devrait être utilisé. } \\
\text { O On ne dispose pas de suffisamment de données sur la } \\
\text { valeur différentielle des différents vaccins antigrippaux } \\
\text { (le CCNI n'a pas effectué d'évaluation du rapport } \\
\text { coût-efficacité) pour formuler des recommandations } \\
\text { comparatives sur le plan des programmes de santé } \\
\text { publique sur l'utilisation des vaccins disponibles. }\end{array}$ \\
\hline
\end{tabular}

Abréviations : CCNI, Comité consultatif national de l'immunisation; HAART, traitement antirétroviral hautement actif; VII, vaccin inactivé contre l'influenza; VII3-Adj, vaccin trivalent inactivé contre I'influenza avec adjuvant; VII3-HD, vaccin trivalent inactivé contre l'influenza à haute dose; VII3-SD, vaccin trivalent inactivé contre l'influenza à dose standard; VII4-cc, vaccin quadrivalent contre

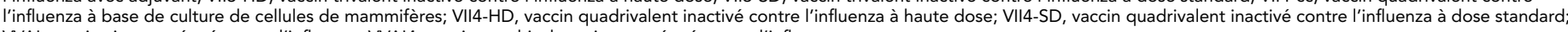
WAI, vaccin vivant atténué contre l'influenza; WAI4, vaccin quadrivalent vivant atténué contre l'influenza

a Les formulations VII3-SD ne pourront pas être utilisées au Canada pendant la saison grippale 2021-2022

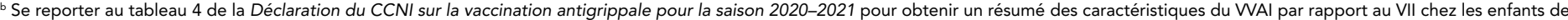
2 à 17 ans

' L'utilisation du VII4-cc est actuellement autorisée chez les adultes et les enfants âgés de 9 ans et plus

' Se reporter au tableau 5 de la Déclaration du CCNI sur la vaccination antigrippale pour la saison 2020-2021 pour obtenir une comparaison des caractéristiques des différents types de vaccins antigrippaux proposés aux adultes de 65 ans et plus (5)

e Les formulations VII3-HD ne pourront pas être utilisées au Canada pendant la saison grippale 2021-2022

Source : Tableau tiré de la Déclaration du CCNI sur la vaccination antigrippale pour la saison 2021-2022 (5) 
Tableau 3 : Posologie et voie d'administration recommandées, selon l'âge, pour les types de vaccins antigrippaux offerts pour la saison 2021-2022

\begin{tabular}{|c|c|c|c|c|c|c|c|}
\hline \multirow{2}{*}{$\begin{array}{l}\text { Groupe } \\
\text { d'âge }\end{array}$} & \multicolumn{6}{|c|}{ Type de vaccin antigrippal (voie d'administration) } & \multirow{2}{*}{$\begin{array}{c}\text { Nombre de } \\
\text { doses requises }\end{array}$} \\
\hline & $\begin{array}{l}\text { VII3-SDa ou } \\
\text { VII4-SDb (IM) }\end{array}$ & VII4-ccc (IM) & VII3-Adjd (IM) & VII3-HDe (IM) & VII4-HDf (IM) & $\begin{array}{c}\text { VVAl4g } \\
\text { (intranasal) }\end{array}$ & \\
\hline 6 à 23 mois & $0,5 \mathrm{~mL}^{\mathrm{h}}$ & - & $0,25 \mathrm{~mL}$ & - & - & - & 1 ou $2^{i}$ \\
\hline 2 à 8 ans & $0,5 \mathrm{~mL}$ & - & - & - & - & $\begin{array}{l}0,2 \mathrm{~mL} \\
(0,1 \mathrm{~mL} \text { par } \\
\text { narine })\end{array}$ & 1 ou $2^{i}$ \\
\hline 9 à 17 ans & $0,5 \mathrm{~mL}$ & $0,5 \mathrm{~mL}$ & - & - & - & $\begin{array}{l}0,2 \mathrm{~mL} \\
(0,1 \mathrm{~mL} \text { par } \\
\text { narine })\end{array}$ & 1 ou $2^{i}$ \\
\hline 18 à 59 ans & $0,5 \mathrm{~mL}$ & $0,5 \mathrm{~mL}$ & - & - & - & $\begin{array}{l}0,2 \mathrm{~mL} \\
(0,1 \mathrm{~mL} \text { par } \\
\text { narine })\end{array}$ & 1 \\
\hline 60 à 64 ans & $0,5 \mathrm{~mL}$ & $0,5 \mathrm{~mL}$ & - & - & - & - & 1 \\
\hline 65 ans et plus & $0,5 \mathrm{~mL}$ & $0,5 \mathrm{~mL}$ & $0,5 \mathrm{~mL}$ & $0,5 \mathrm{~mL}$ & $0,7 \mathrm{~mL}$ & - & 1 \\
\hline 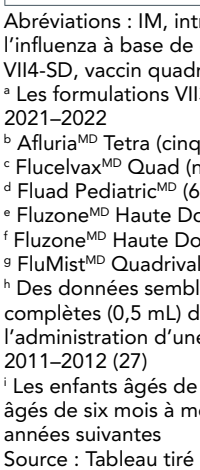 & $\begin{array}{l}\text { musculaire; VII3-Adj, vac } \\
\text { ulture de cellules de mam } \\
\text { alent inactivé contre l'inf } \\
\text { SD (AgrifluMD [six mois et } \\
\text { ns et plus), Flulaval'MD Tet } \\
\text { uf ans et plus) } \\
23 \text { mois) ou FluadMD (65 } \\
\text { ( } 65 \text { ans et plus) était pr } \\
\text { Quadrivalent ( } 65 \text { ans et } \\
\text { th ( } 2 \text { à } 59 \text { ans) } \\
\text { tt indiquer une améliorat } \\
\text { vaccin inactivé contre l'in } \\
\text { dose complète du vaccin } \\
\text { x mois à moins de neuf a } \\
\text { ns de neuf ans qui ont de } \\
\text { la Déclaration du CCNI }\end{array}$ & $\begin{array}{l}\text { n trivalent inactivé cont } \\
\text { nifères; VII4-HD, vaccin } \\
\text { uenza à dose standard; } \\
\text { olus], FluviralMD [six mois } \\
\text { (six mois et plus), Fluz } \\
\text { ans et plus) } \\
\text { cédemment autorisé, m } \\
\text { lus) } \\
\text { on modérée de la répon } \\
\text { luenza sans adjuvant ( } 25 \\
\text { nactivé sans adjuvant ch } \\
\text { s qui n'ont jamais été v } \\
\text { à reçu, comme il se doit } \\
\text { ur la vaccination antigri }\end{array}$ & $\begin{array}{l}\text { e l'influenza avec adjuva } \\
\text { quadrivalent inactivé con } \\
\text { VAI4, vaccin quadrivalen } \\
\text { et plus] et Influvac }{ }^{\mathrm{MD}} \text { [tro } \\
\mathrm{ne}^{\mathrm{MD}} \text { Quadrivalent (six n } \\
\text { ais la commercialisation } \\
\text { se immunitaire chez les n } \\
\text {,26). Cette amélioration } \\
\text { ez les sujets de tous âge } \\
\text { uccinés contre la grippe s } \\
\text { une dose complète ou } \\
\text { opale pour la saison } 202\end{array}$ & $\begin{array}{l}\text { t; VII3-HD, vaccin trivale } \\
\text { re l'influenza à haute do } \\
\text { vivant atténué contre l'i } \\
\text { s ans et plus]) sont autor } \\
\text { ois et plus), Influvac }{ }^{\mathrm{MD}} \mathrm{Te} \\
\text { u vaccin a été interromp } \\
\text { uurrissons, sans qu'il y ait } \\
\text { nodérée de la réponse a } \\
\text {. Pour plus de renseigne } \\
\text { aisonnière doivent recev } \\
\text { lus du vaccin antigrippal } \\
\text {-2022 (5) }\end{array}$ & $\begin{array}{l}\text { ti inactivé contre l'influer } \\
\text { e; VII3-SD, vaccin trivale } \\
\text { fluenza; -, ne s'applique } \\
\text { sées mais ne pourront p } \\
\text { ra (trois ans et plus) } \\
\text { e à partir de février } 202 \\
\text { augmentation de la réac } \\
\text { ticorps, sans augmentat } \\
\text { nents, voir la Déclaratior } \\
\text { ir deux doses du vaccin, } \\
\text { saisonnier dans le passé }\end{array}$ & $\begin{array}{l}\text { nza à haute dose; VII4-cc, } \\
\text { nt inactivé contre l'influer } \\
\text { e pas } \\
\text { as être utilisées au Canad } \\
1 \\
\text { ctogénicité, après l'admin } \\
\text { tion de la réactogénicité, } \\
\text { n sur la vaccination antigr } \\
\text { à au moins quatre semai } \\
\text { devraient recevoir une d }\end{array}$ & $\begin{array}{l}\text { accin quadrivalent contre } \\
\text { pendant dose standard; } \\
\text { pendaison grippale } \\
\text { stration de doses } \\
\text { st la raison qui justifie } \\
\text { ppale pour la saison } \\
\text { es d'intervalle. Les enfants } \\
\text { se par saison, au cours des }\end{array}$ \\
\hline
\end{tabular}

\section{Conclusion}

Le CCNI continue de recommander la vaccination antigrippale annuelle pour toutes les personnes de six mois et plus (à condition de connaître les indications et les contre-indications relatives à l'âge propres à chaque produit), en accordant une importance particulière aux personnes à risque élevé de complications ou d'hospitalisation liées à la grippe. Pour la saison grippale 2021-2022, le CCNI recommande pour la première fois qu'Influvac Tetra et Flucelvax Quad puissent être considérés comme des options parmi les vaccins inactivés quadrivalents contre l'influenza proposés aux adultes et aux enfants pour leur vaccination annuelle. Le CCNI recommande également que Fluzone Haute Dose Quadrivalent soit considéré comme une option pour les adultes âgés de 65 ans et plus.

De plus, l'administration du vaccin antigrippal est particulièrement recommandée aux personnes susceptibles de transmettre la grippe à des sujets à risque élevé, aux personnes qui fournissent des services communautaires essentiels, et aux personnes en contact direct avec de la volaille infectée par le virus de la grippe aviaire durant les opérations d'abattage.

\section{Déclaration des auteurs}

A. S. - Rédaction de la version préliminaire, révision et édition

K. Y. - Révision et édition

R. H. - Révision et édition

Le document Chapitre sur la grippe du Guide canadien d'immunisation et Déclaration du CCNI sur la vaccination antigrippale pour la saison 2021-2022 a été préparé par K. Young, L. Zhao, A. Sinilaite, R. Stirling et R. Harrison, au nom du Groupe de travail sur l'influenza du CCNI et a été approuvé par le CCNI.

\section{Intérêts concurrents}

Aucun.

\section{Remerciements}

Membres du Groupe de travail sur l'influenza : R. Harrison (présidente), I. Gemmill, K. Klein, D. Kumar, J. Langley, J. McElhaney, A. McGeer, D. Moore et B. Warshawsky.

Anciens membres : N. Dayneka, S. Smith 
Membres du CCNI : S. Deeks (présidente), R. Harrison (vice-présidente), J. Bettinger, P. De Wals, E. Dubé, V. Dubey, K. Hildebrand, K. Klein, J. Papenburg, C. Rotstein, B. Sander, S. Smith et S. Wilson.

Anciens membres : C. Quach (présidente), N. Dayneka, S. Gantt

Représentants de liaison : L. M. Bucci (Association canadienne de santé publique), E. Castillo (Société des obstétriciens et gynécologues du Canada), A. Cohn (Centers for Disease Control and Prevention, États-Unis), L. Dupuis (Association des infirmières et infirmiers du Canada), J. Emili (Collège des médecins de famille du Canada), D. Fell (Association canadienne pour la recherche, l'évaluation et l'éducation en immunisation), M. Lavoie (Conseil des médecins hygiénistes en chef), D. Moore (Société canadienne de pédiatrie), M. Naus (Comité canadien sur l'immunisation) et A. Pham-Huy (Association pour la microbiologie médicale et l'infectiologie Canada).

Anciens représentants d'office : D. Danoff (Direction des produits de santé commercialisés, Santé Canada [SC]), E. Henry (Centre de l'immunisation et des maladies respiratoires infectieuses [CIMRI], Agence de la santé publique du Canada [l'Agence]), M. Lacroix (Groupe consultatif d'éthique en santé publique, I'Agence), J. Pennock (CIMRI, I'Agence), R. Pless (Direction des produits biologiques et des thérapies génétiques, $\mathrm{SC}), \mathrm{G}$. Poliquin (Laboratoire national de microbiologie,

l'Agence), V. Beswick-Escanlar (Défense nationale et Forces armées canadiennes) et T. Wong (Direction générale de la santé des Premières nations et des Inuits, Services aux Autochtones Canada).

Le Comité consultatif national de l'immunisation reconnaît et apprécie la contribution de A. House, M. Laplante, C. Tremblay et $\mathrm{M}$. Tunis à cette déclaration.

\section{Financement}

Les travaux du Comité consultatif national de l'immunisation bénéficient du soutien de l'Agence de la santé publique du Canada.

\section{Références}

1. Organisation mondiale de la Santé. Principaux repères : Grippe (saisonnière). Genève (CH) : OMS; 2014. https://www.who.int/fr/news-room/fact-sheets/detail/ influenza-(seasonal)

2. World Health Organization. Tools and toolkits: FluNet. Geneva (CH): WHO; 2021. https://www.who.int/tools/flunet

3. Agence de la santé publique du Canada. Grippe (influenza) : Surveillance ÉpiGrippe. Ottawa (ON) : ASPC; 2020. https:// www.canada.ca/fr/sante-publique/services/maladies/grippeinfluenza/surveillance-influenza.html
4. Ismail SJ, Hardy K, Tunis MC, Young K, Sicard N, Quach C. A framework for the systematic consideration of ethics, equity, feasibility, and acceptability in vaccine program recommendations. Vaccine 2020;38(36):5861-76. DOI PubMed

5. Comité consultatif national de l'immunisation. Chapitre sur la grippe du Guide canadien d'immunisation et Déclaration sur la vaccination antigrippale pour la saison 2021-2022. Ottawa (ON) : ASPC; 2021. https://www.canada.ca/fr/ sante-publique/services/publications/vaccins-immunisation/ guide-canadien-immunisation-declaration-vaccinationantigrippale-2021-2022.html

6. Comité consultatif national de l'immunisation.

Recommandations pour l'immunisation fondées sur des données probantes - Méthodes du Comité consultatif national de l'immunisation. Relevé des maladies transmissibles au Canada. 2009;35(ACS-1):1-10.

https://www.canada.ca/fr/sante-publique/services/rapportspublications/releve-maladies-transmissibles-canada-rmtc/ numero-mensuel/2009-35/methodes-comite-consultatifnational-immunisation.html

7. Comité consultatif national de l'immunisation. Déclaration supplémentaire - Vaccins antigrippaux issus de cultures cellulaires de mammifères. Ottawa (ON) : ASPC; 2020. https://www.canada.ca/fr/sante-publique/services/ immunisation/comite-consultatif-national-immunisation-ccni/ vaccins-antigrippaux-issus-cultures-cellulaires-mammiferes. html

8. Harris RP, Helfand M, Woolf SH, Lohr KN, Mulrow CD, Teutsch SM, Atkins D; Methods Work Group, Third US Preventive Services Task Force. Current methods of the US Preventive Services Task Force: a review of the process. Am J Prev Med 2001;20(3 Suppl):21-35. DOI PubMed

9. Comité consultatif national de l'immunisation. Lignes directrices sur l'administration de vaccins contre la grippe en présence de la COVID-19. Ottawa (ON) : ASPC; 2020. https://www.canada.ca/fr/sante-publique/services/ immunisation/comite-consultatif-national-immunisation-ccni/ lignes-directrices-administration-vaccins-contre-grippecovid-19.html

10. Agence de la santé publique du Canada. Lignes directrices sur l'utilisation de vaccins contre la grippe en présence de la COVID-19. https://www.canada.ca/fr/sante-publique/ services/immunisation/comite-consultatif-nationalimmunisation-ccni/lignes-directrices-utilisation-vaccinscontre-grippe-covid-19.html

11. Boikos C, Sylvester G, Sampalis J, Mansi J. Effectiveness of the Cell Culture- and Egg-Derived, Seasonal Influenza Vaccine during the 2017-2018 Northern Hemisphere Influenza Season. Canadian Immunization Conference. 2018. 2018 Dec 04-06; Ottawa, ON, Canada [poster presentation]. https://www.izsummitpartners.org/content/ uploads/2019/05/1-effectiveness-of-cell-culture-and-eggderived-flu-vax-during-2017-2018-flu-season.pdf

12. DeMarcus L, Shoubaki L, Federinko S. Comparing influenza vaccine effectiveness between cell-derived and egg-derived vaccines, 2017-2018 influenza season. Vaccine 2019;37(30):4015-21. DOI PubMed 
13. Izurieta HS, Chillarige $Y$, Kelman J, Wei Y, Lu Y, Xu W, Lu M, Pratt D, Chu S, Wernecke M. MaCurdy T, Forshee R. Relative effectiveness of cell-cultured and egg-based influenza vaccines among elderly persons in the United States, 2017-18, 2017-18. J Infect Dis 2019;220(8):1255-64. DOI PubMed

14. Klein NP, Fireman B, Goddard K, Zerbo O, Asher J, Zhou J, King J, Lewis N. LB15. Vaccine Effectiveness of Flucelvax Relative to Inactivated Influenza Vaccine During the 2017-18 Influenza Season in Northern California. Open Forum Infect Dis. 2018;5(Suppl 1):S764. DOI

15. Bart S, Cannon K, Herrington D, Mills R, Forleo-Neto E, Lindert K, Abdul Mateen A. Immunogenicity and safety of a cell culture-based quadrivalent influenza vaccine in adults: A Phase III, double-blind, multicenter, randomized, non-inferiority study. Hum Vaccin Immunother 2016;12(9):2278-88. DOI PubMed

16. Hartvickson R, Cruz M, Ervin J, Brandon D, Forleo-Neto E, Dagnew AF, Chandra R, Lindert K, Mateen AA. Non-inferiority of mammalian cell-derived quadrivalent subunit influenza virus vaccines compared to trivalent subunit influenza virus vaccines in healthy children: a phase III randomized, multicenter, double-blind clinical trial. Int J Infect Dis 2015;41:65-72. DOl PubMed

17. Frey S, Vesikari T, Szymczakiewicz-Multanowska A, Lattanzi M, Izu A, Groth N, Holmes S. Clinical efficacy of cell culture-derived and egg-derived inactivated subunit influenza vaccines in healthy adults. Clin Infect Dis 2010;51(9):997-1004. DOI PubMed

18. Vesikari T, Block SL, Guerra F, Lattanzi M, Holmes S, Izu A, Gaitatzis N, Hilbert AK, Groth N. Immunogenicity, safety and reactogenicity of a mammalian cell-culturederived influenza vaccine in healthy children and adolescents three to seventeen years of age. Pediatr Infect Dis J 2012;31(5):494-500. DOI PubMed

19. Ambrozaitis A, Groth N, Bugarini R, Sparacio V, Podda A, Lattanzi M. A novel mammalian cell-culture technique for consistent production of a well-tolerated and immunogenic trivalent subunit influenza vaccine. Vaccine 2009;27(43):6022-9. DOI PubMed

20. Szymczakiewicz-Multanowska A, Groth N, Bugarini R, Lattanzi M, Casula D, Hilbert A, Tsai T, Podda A. Safety and immunogenicity of a novel influenza subunit vaccine produced in mammalian cell culture. J Infect Dis 2009;200(6):841-8. DOI PubMed
21. Loebermann M, Fritzsche $C$, Geerdes-Fenge $H$, Heijnen $E$, Kirby D, Reisinger EC. A phase III, open-label, single-arm, study to evaluate the safety and immunogenicity of a trivalent, surface antigen inactivated subunit influenza virus vaccine produced in mammalian cell culture (Optaflu ${ }^{\circledR}$ ) in healthy adults. Infection 2019;47(1):105-9. DOI PubMed

22. Nolan T, Chotpitayasunondh T, Capeding MR, Carson S, Senders SD, Jaehnig P, de Rooij R, Chandra R. Safety and tolerability of a cell culture derived trivalent subunit inactivated influenza vaccine administered to healthy children and adolescents: A Phase III, randomized, multicenter, observer-blind study. Vaccine 2016;34(2):230-6. DOI PubMed

23. Seqirus UK. Limited. Product monograph: Flucelvax ${ }^{\circledR}$ QUAD: Influenza Vaccine (surface antigen, inactivated, prepared in cell cultures). 2021. https://www.seqirus.ca/-/media/ seqirus-canada/docs-en/flucelvax-quad-ca-pm-2-approved8mar2021.pdf?la=en-us\&hash=9504E4305DF163072338F3C 307640B7379230DC8

24. Agence de la santé publique du Canada. Guide canadien d'immunisation : Partie 3 - Vaccination de populations particulières. Ottawa (ON) : ASPC; 2015. https://www. canada.ca/fr/sante-publique/services/publications/viesaine/guide-canadien-immunisation-partie-3-vaccinationpopulations-particulieres.html

25. Langley JM, Vanderkooi OG, Garfield HA, Hebert J, Chandrasekaran V, Jain VK, Fries L. Immunogenicity and safety of 2 dose levels of a thimerosal-free trivalent seasonal influenza vaccine in children aged 6-35 months: a randomized, controlled trial. J Pediatric Infect Dis Soc 2012;1(1):55-63. DOI PubMed

26. Skowronski DM, Hottes TS, Chong M, De Serres G, Scheifele DW, Ward BJ, Halperin SA, Janjua NZ, Chan T, Sabaiduc S, Petric M. Randomized controlled trial of dose response to influenza vaccine in children aged 6 to 23 months. Pediatrics 2011;128(2):e276-89. DOl PubMed

27. Comité consultatif national de l'immunisation (CCNI). Déclaration sur la vaccination antigrippale pour la saison 2011-2012. Relevé des maladies transmissibles au Canada 2011;37(ACS-5):1-61. DOI 


\section{Annexe}

Tableau A1 : Classement de l'importance des recommandations du Comité consultatif national de l'immunisation (CCNI) et de la qualité des données probantes

\section{Importance de la recommandation du \\ CCNI \\ basée sur des facteurs non isolés de la validité des preuves (e.g. le besoin en santé publique)}

Libellé

\section{Justification}

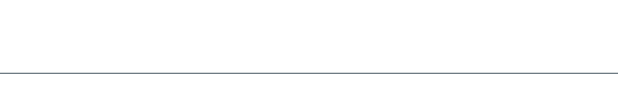

Implication

的

Qualité des données

selon l'évaluation de l'ensemble des données
" doit/ne doit pas être proposé »

Les avantages connus/anticipés l'emportent sur les inconvénients connus/anticipés (" doit »),

OU les inconvénients connus/anticipés l'emportent sur les avantages connus/anticipés (« ne doit pas »)

Une recommandation forte s'applique à la plupart des populations/personnes et doit être suivie à moins qu'il n'existe une justification claire et convaincante pour une autre approche

A : données probantes pour recommander

$B$ : données suffisantes pour recommander

$C$ : données contradictoires, mais d'autres facteurs peuvent avoir une incidence sur la prise de décision

$D$ : données suffisantes pour ne pas recommander

E : données probantes pour déconseiller

I: données insuffisantes (en qualité ou en quantité), mais d'autres facteurs peuvent avoir une incidence sur la prise de décision
" peut être considéré »

Les avantages connus/anticipés sont étroitement équilibrés avec les inconvénients connus/anticipés,

$\mathrm{OU}$ il existe une incertitude quant aux preuves des avantages et des inconvénients

Une recommandation discrétionnaire peut être envisagée pour certaines populations/ personnes dans certaines circonstances

Des approches alternatives peuvent être considéré

\section{Discrétionnaire}

